\title{
Risk Evaluation of Secondary Cancer Induced by Breast Cancer Radiotherapy Treatment
}

Ibrahim Duhaini ${ }^{*}{ }^{*}$ Fatima Hijazi $^{2}$, Eva Sabbah ${ }^{3}$, Saad Ayoubi ${ }^{1}$, Sadek Nehmeh ${ }^{4}$, Ahmad Marouf ${ }^{5}$ and Mahmoud Korek ${ }^{5}$

${ }^{1}$ Rafik Hariri University Hospital, Beirut, Lebanon

${ }^{2}$ Labib Medical Center, Saida, Lebanon

${ }^{3}$ Raee Hospital, Saida, Lebanon

${ }^{4}$ Weill Cornell Medicine, New York, USA

${ }^{5}$ Beirut Arab University, Beirut, Lebanon

\begin{abstract}
The Radiotherapy technologies are one of the most common treatments for cancer, they have been successfully used to damage cancer cells, with as little harm as possible to nearby healthy cells, however Secondary cancer risk following radiotherapy is an increasingly important topic in clinical oncology with impact on treatment decision making and on patient management. The paper aims to investigate and evaluate the current practice in radiotherapy through its different phases and to quantify the risk of secondary primary cancer following radiotherapy in breast cancer by dose calculation and risk estimation of second cancer; to make recommendations for a safer practice in radiotherapy department. The data collected from NBGUH and RHUH with dosimetry calculations on retrospective female patient's files and verifies with dose measurements on RANDO Phantom.
\end{abstract}

The results showed $85 \%$ of compliance in radiotherapy safe practices, calculated Probability of Causation after breast radiotherapy for contralateral and Ipsilateral breast cancer $0.17 \%$, and for lung is $32 \%$, for spinal cord is $24 \%$. While the PC in phantom measurements in Ipsilateral lung $43.22 \%$ and PC for contralateral lung $3.099 \%$.

Keywords: Radiotherapy; Breast cancer; Oncology; Radiation

\section{Introduction}

The Breast radiotherapy is used to treat female patients in purpose to shrink the tumor or to eliminate the risk of cancer recurrence. It is also known that breast radiotherapy may have side effects to organs at risk nearby the treated breast. This paper highlights the risk of second primary cancer induced by breast radiotherapy by evaluation and estimation of this risk using dosimetry measurements and calculations for some Organs at Risk and performing survey assessment of healthcare professional compliance during radiotherapy process.

The overall risk for developing a radiation related malignancy has historically been best described among breast cancer patients. Breast cancer is a disease in which many patients undergo treatment with radiation, and fortunately, most breast cancer patients are cured of their disease and live for many years after treatment. During radiation therapy of the breast, other organs in the body may receive a significant radiation dose that triggers a secondary cancer in these organs. In this study, the out of field radiation doses measured in some organs and evaluated for the development of radiation induced cancer as recently defined by the International Commission on Radiological protection (ICRP2003) [1-3].

In the past, breast radiotherapy has been used mainly in conjunction with extensive surgery to treat primary breast cancer. Today it is common to treat early disease with local excision and irradiation of the breast. It is conceivable that scatter or incidental exposure to the contralateral breast, lung, and other OAR which can amount to a dose of several grays, contributes to the overall risk of a second cancer. On the other hand, practically all epidemiologic studies indicate that radiogenic breast cancer is exceedingly rare among women who have undergone irradiation after 40 years of age. To evaluate the risk of second primary cancer in relation to radiotherapy and age at exposure, we undertook a retrospective case study of women in two hospitals in Lebanon namely Nabih Berry Governmental University Hospital (NBGUH) and Rafik Hariri University Hospital (RHUH). For women who underwent breast irradiation, the risk of a second primary cancer for OAR was estimated as a function of the radiation dose to nearby organs $[4,5]$.

\section{Material and Method}

Assessing the relative risk of secondary cancer after breast radiotherapy and evaluating the current practice in radiotherapy department in NBGUH and RHUH was performed. A summary analysis of the quality of the organizations' performance in delivering the radiotherapy as a safe medical treatment was conducted to estimate the risk and the Probability of Causation (PC) for second malignancy.

\section{Hospital level audit}

A hospital-level audit survey tool was used to assess the internal radiotherapy process, staff compliance, the professional practice during the radiation therapy phases, and evaluation of the safe practice in radiation therapy process in NBGUH.

Radiation dose calculation for organs at risk (OAR): A retrospective data collection on patient's medical record was performed to evaluate the radiation therapy induced second primary cancers including age at exposure, medical and family history of cancer, the radiation therapy modalities used and the doses of planning total volume of the irradiation dose, the mean dose of radiation to the organs at risk like the heart, the spinal cord, and the oesophagus in

*Corresponding author: Ibrahim Duhaini, Rafik Hariri University Hospital, Beirut, Lebanon, Tel: +9611830000; E-mail: duhaini@yahoo.com

Received November 02, 2017; Accepted November 06, 2017; Published November 15, 2017

Citation: Duhaini I, Hijazi F, Sabbah E, Ayoubi S, Nehmeh S, et al. (2017) Risk Evaluation of Secondary Cancer Induced by Breast Cancer Radiotherapy Treatment. J Nucl Med Radiat Ther 8: 346. doi: 10.4172/2155-9619.1000346

Copyright: $\odot 2017$ Duhaini I, et al. This is an open-access article distributed under the terms of the Creative Commons Attribution License, which permits unrestricted use, distribution, and reproduction in any medium, provided the original author and source are credited. 
Citation: Duhaini I, Hijazi F, Sabbah E, Ayoubi S, Nehmeh S, et al. (2017) Risk Evaluation of Secondary Cancer Induced by Breast Cancer Radiotherapy Treatment. J Nucl Med Radiat Ther 8: 346. doi: 10.4172/2155-9619.1000346

Page 2 of 5

both NBGUH and RHUH. The effective dose was calculated using IAEA equations. The risk estimation and probability of causation for second primary cancer to OAR was derived from ICRP equations based on BEIR V Reports according to the following equation:

Risk estimation $=1+\alpha_{1} D \exp \left(\beta_{2} \log _{e}(t / 20)+\beta_{3}\left[\log _{e}\left(\frac{t}{20}\right)\right]^{2}+\beta_{4}[\mathrm{e}-15]\right)$

Where:

- $\beta 2=-0.104, \beta 3=-2.212$, and $\beta 4=-0.0 .628$

- $\mathrm{e}=$ age at exposure, $\mathrm{t}=$ time since exposure (in this study $\mathrm{t}=25$ for all the patients)

- $\mathrm{D}$ is the mean effective dose in $(\mathrm{Sv})$

The probability of causations (PC) was also calculated:

$\mathrm{PC}=\frac{\mathrm{RE}-1}{\mathrm{RE}}$

Dose assessments and measurements using RANDO phantom with GafChromic films: Gafchromic EBT3 films were used as dosimeters on RANDO phantom for a standard breast radiotherapy treatment and Treatment Planning dose calculations were done for comparison. The measurements were done at The National Center of Cancer and Research in Doha-Qatar. Measurements, simulation and dose calculation were performed on female phantom as shown in Figure 1. The estimation of radiation induced second cancer to the lungs was conducted using BEIR V Risk Model and Equations.

\section{Results}

The results originated from collecting data using Audit survey forms and the planning system data from NBGUH and RHUH showed $85 \%$ compliance of health care professionals during all stages of radiotherapy process. The result of bivariate correlation in RHUH showed that there is a negative relationship between age at exposure and the risk of second primary breast cancer and a weak correlation between dose received to contralateral breast and the risk of second primary breast cancer. While at NBGUH the result of bivariate correlation showed that there is a weak correlation between age at exposure and probability of causation of second cancer on spinal cord level, and there is a weak negative correlation between the age of patient at exposure and the risk of second primary lung cancer.

The dose-volume for OAR as heart, spinal cord, lung and contralateral breast were calculated to all observed female patients in NBGUH and RHUH the results are shown in Table 1.

Surface skin dose for the left breast is, and point doses of the lungs are measured and compared with the calculated ones. The calculated and measured doses are presented as percent of prescribed dose, see Table 2 .

The calculated RR and PC values of the lungs -both Ipsilateral and contralateral- and their average values are represented in Table 3 along with the mean and effective doses of the lungs.

The Pinnacle 3 treatment planning system provides a comprehensive set of tools for setting up and evaluating treatment plans. The software includes options for simulation, photon, electron, stereotactic radiosurgery, proton, and brachytherapy treatment planning. For photon external beam treatment plans, Pinnacle 3 uses a 3D convolution superposition dose calculation algorithm. This model computes the dose from first principles and uses a limited set of measurements to fit the model to the measured data, see Figure 2.

The phantom measurements showed the average Cancer Probability of Causation in the Ipsilateral Lung to be $43.2 \%$ and that for Contralateral Lung was 3.099\%.
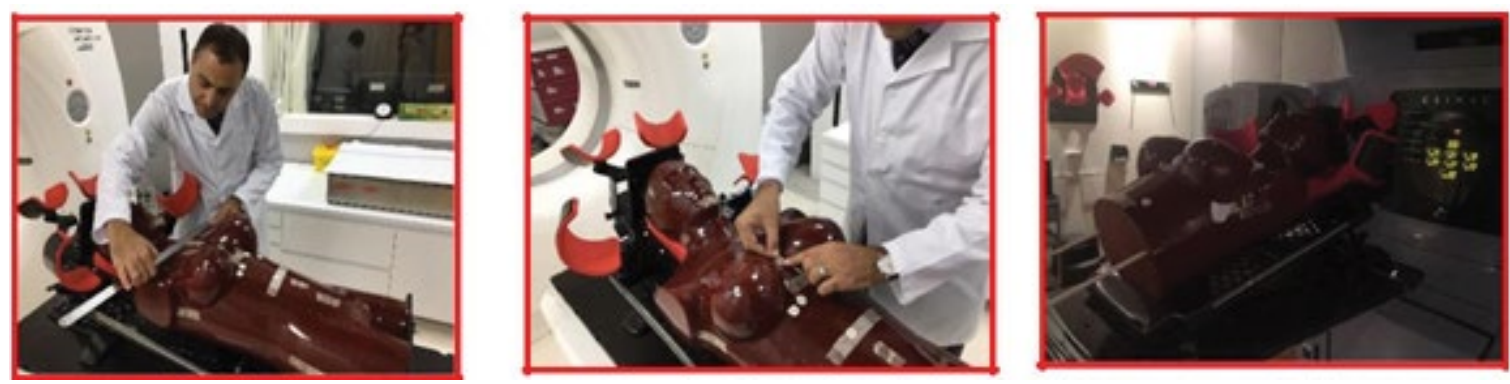

Figure 1: Female rando phantom simulation in the nccr in doha-qatar.

\begin{tabular}{|c|c|c|c|c|c|}
\hline \multirow{2}{*}{ Variables } & \multicolumn{2}{|c|}{ Age at exposure } & Spinal Cord & Contralateral breast & Lung \\
\cline { 2 - 6 } & NBGUH & RHUH & & & \\
\hline Average & 49.5 & 51.5 & $2.9 \mathrm{cGy}$ & $23.3 \mathrm{cGy}$ & $1.7 \mathrm{cGy}$ \\
\hline Max & 78 & 70 & $9 \mathrm{cGy}$ & $0.7 \mathrm{cGy}$ & $38.8 \mathrm{cGy}$. \\
\hline
\end{tabular}

Table 1: Measured and calculated dose volume of oar and the age at exposure.

\begin{tabular}{|c|c|c|c|c|c|}
\hline \multicolumn{6}{|c|}{ Dose (\% of prescribed dose) } \\
\hline Variables & Left breast $^{\mathrm{a}}$ & \multicolumn{2}{|c|}{ Ipsilateral lung } & \multicolumn{2}{|c|}{ Contralateral lung } \\
\hline Position & & Superior medial & Superior lateral & Superior medial & Superior lateral \\
\hline Measured & 85.5 & 7 & 105 & 4.5 & 6.5 \\
\hline Calculated & 84 & 6.3 & 90 & 3.7 & 4.9 \\
\hline
\end{tabular}

Table 2: Measured and calculated surface skin dose of left breast, and point doses of ipsilateral and contralateral lung. 
Citation: Duhaini I, Hijazi F, Sabbah E, Ayoubi S, Nehmeh S, et al. (2017) Risk Evaluation of Secondary Cancer Induced by Breast Cancer Radiotherapy Treatment. J Nucl Med Radiat Ther 8: 346. doi: 10.4172/2155-9619.1000346

Page 3 of 5

\begin{tabular}{|c|c|c|c|c|c|c|c|c|}
\hline \multirow{2}{*}{$\begin{array}{l}\text { Variables } \\
\text { Patient ID }\end{array}$} & \multicolumn{4}{|c|}{ Ipsilateral Lung } & \multicolumn{4}{|c|}{ Contralateral Lung } \\
\hline & Mean dose (cGy) & $\begin{array}{c}\text { Effective dose } \\
\text { (Sv) }\end{array}$ & RR & PC (\%) & $\begin{array}{l}\text { Mean dose } \\
\text { (cGy) }\end{array}$ & $\begin{array}{c}\text { Effective dose } \\
\text { (Sv) }\end{array}$ & $\mathbf{R R}$ & PC (\%) \\
\hline 1 & 538.8 & 0.646 & 1.608 & 37.79 & 29.3 & 0.035 & 1.033 & 3.15 \\
\hline 2 & 966.2 & 1.159 & 2.089 & 52.13 & 54.7 & 0.066 & 1.062 & 5.8 \\
\hline 3 & 1178.1 & 1.414 & 2.328 & 57.05 & 33.1 & 0.04 & 1.037 & 3.59 \\
\hline 4 & 310.8 & 0.372 & 1.35 & 25.9 & 22.9 & 0.027 & 1.026 & 2.15 \\
\hline 5 & 429.8 & 0.515 & 1.485 & 32.64 & 14.7 & 0.018 & 1.017 & 1.62 \\
\hline 6 & 382 & 0.458 & 1.431 & 30.1 & 18.1 & 0.022 & 1.02 & 1.99 \\
\hline 7 & 1050.5 & 1.26 & 2.184 & 54.22 & 37.3 & 0.044 & 1.042 & 4.03 \\
\hline 8 & 362.5 & 0.435 & 1.409 & 29 & 11 & 0.013 & 1.012 & 1.22 \\
\hline 9 & 1276.6 & 1.531 & 2.439 & 59 & 40.7 & 0.048 & 1.046 & 4.37 \\
\hline 10 & 768.3 & 0.921 & 1.866 & 46.41 & 33.8 & 0.041 & 1.038 & 3.67 \\
\hline 11 & 1100.3 & 1.32 & 2.241 & 55.36 & 30.6 & 0.036 & 1.035 & 3.33 \\
\hline 12 & 415.6 & 0.498 & 1.469 & 31.6 & 23.9 & 0.028 & 1.027 & 2.16 \\
\hline 13 & 361.7 & 0.434 & 1.408 & 28.96 & 24.4 & 0.029 & 1.028 & 2.67 \\
\hline 14 & 800.8 & 0.96 & 1.903 & 47.44 & 30.9 & 0.037 & 1.035 & 3.62 \\
\hline 15 & 793.1 & 0.951 & 1.894 & 47.2 & 32.3 & 0.038 & 1.036 & 3.51 \\
\hline 16 & 852.6 & 1.023 & 1.961 & 49 & 27.3 & 0.032 & 1.031 & 2.97 \\
\hline 17 & 929.2 & 1.115 & 2.048 & 51.16 & 30.8 & 0.036 & 1.035 & 3.35 \\
\hline 18 & 332.7 & 0.399 & 1.375 & 27.27 & 15.9 & 0.019 & 1.018 & 1.75 \\
\hline 19 & 725 & 0.87 & 1.817 & 44.97 & 29.4 & 0.035 & 1.033 & 3 \\
\hline 20 & 785.9 & 0.943 & 1.886 & 46.98 & 33.1 & 0.039 & 1.037 & 3.59 \\
\hline 21 & 1022.1 & 1.226 & 2.152 & 53.54 & 32.8 & 0.039 & 1.037 & 3.55 \\
\hline Average & 732.5 & 0.878 & 1.826 & 43.22 & 28.9 & 0.034 & 1.032 & 3.099 \\
\hline
\end{tabular}

Table 3: Mean dose, effective dose, rr, and pc values of ipsilateral and contralateral lungs.
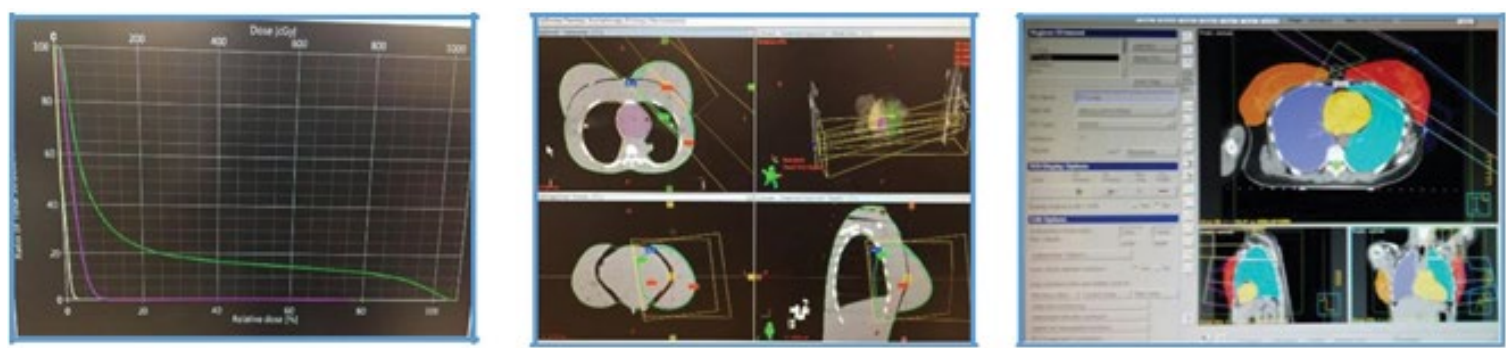

Figure 2: Phantom treatment planning using tps.

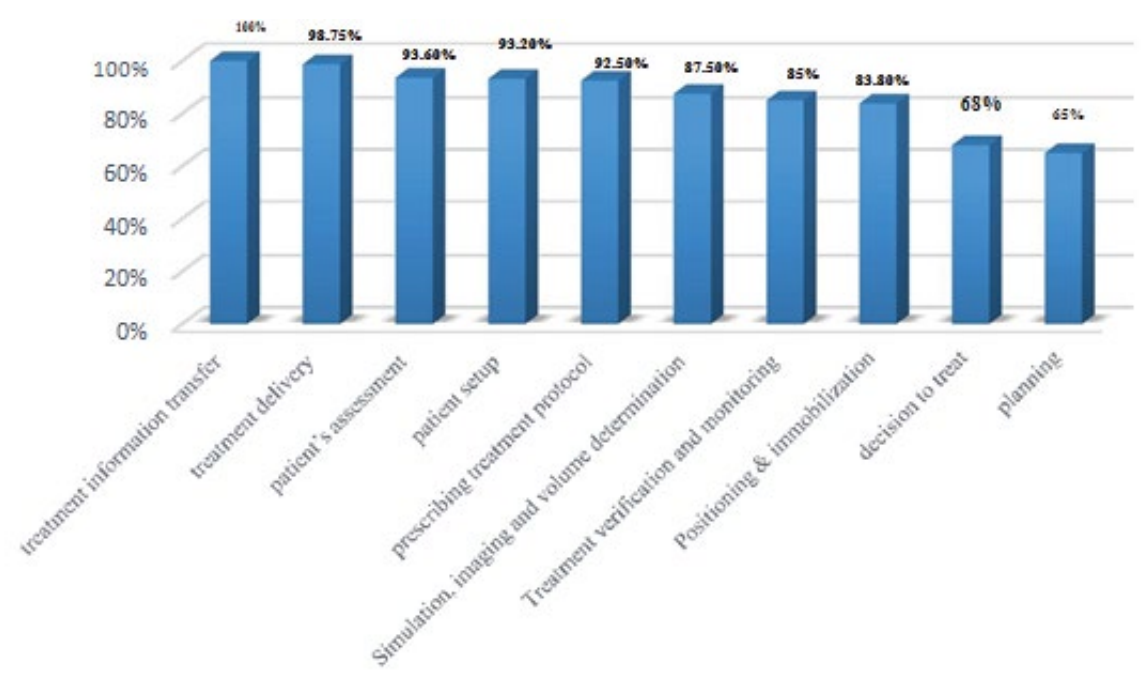

Figure 3: Percentage of compliance in Radiotherapy process. 


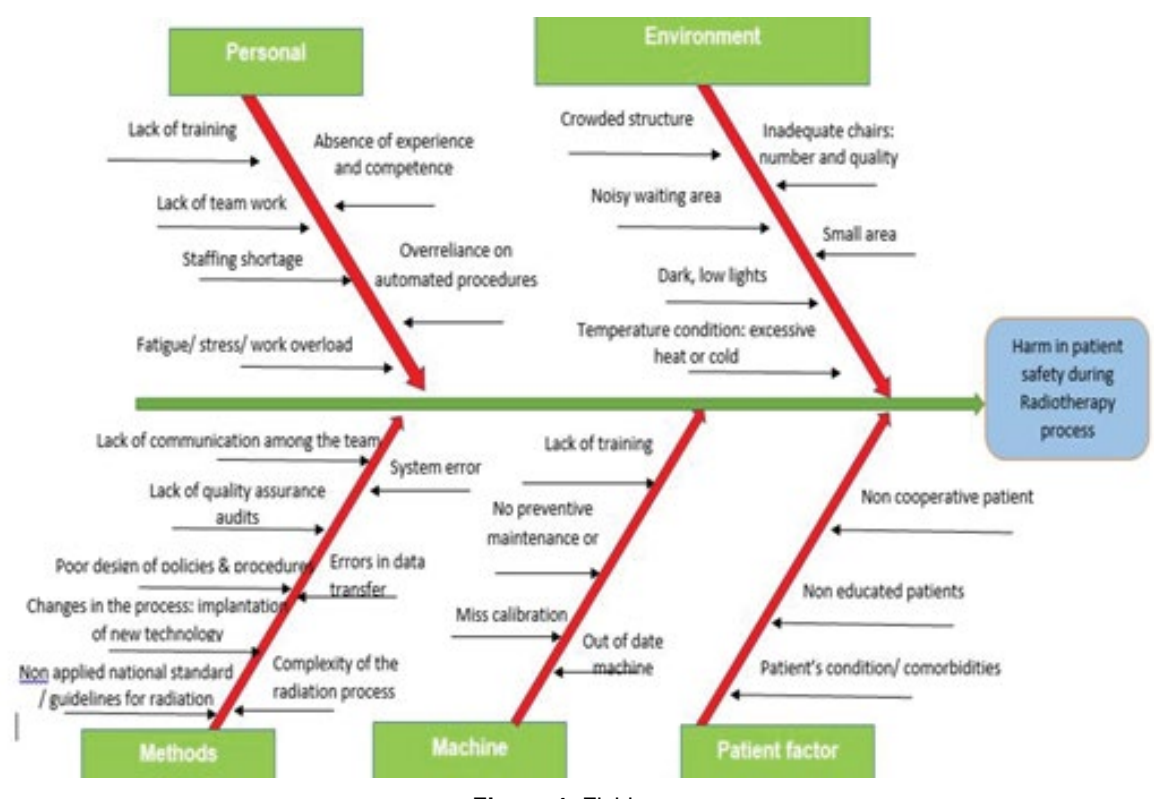

Figure 4: Fishbone.

\section{Discussion}

The Current treatment for early-stage breast cancer couples conservative surgery (also called lumpectomy, quadrantectomy, and partial mastectomy) with radiotherapy in a cumulative dose of approximately $50 \mathrm{~Gy}$ to the whole breast. The dose of radiation delivered to the contralateral breast and lung can be in several Grays. The risk associated with radiation was significantly low. Radiotherapy did not contribute to the high risk of second cancer (contralateral, Ipsilateral breast, spinal cord) among women whose first cancers occurred after they reached 40 years of age. Given the general awareness that radiation can induce as well as cure breast cancer, it is reassuring that our data indicate a low risk of a second cancer after doses of several Grays to the opposite breast. The risk estimation of second primary cancer show a moderate relationship with age at exposure and the dose delivered to female breast patients. The calculated risk estimation after dose measurements for female RANDO phantom using the same data to female breast patients in RHUH and NBGUH showed the mean of probability of causation (PC) of contralateral lung (3.099\%) is lower than that of Ipsilateral lung (43.22\%). This is obvious because the dose to the Ipsilateral lung is higher than that of the contralateral lung, so as the relative risk.

Calculated PC of lungs showed strong correlation with dose; $\mathrm{r}=0.989, \mathrm{p}<0.01$ for Ipsilateral lung and $\mathrm{r}=0.991, \mathrm{p}<0.01$ for contralateral lung. However the means of PC for both lungs are below $50 \%$. This means that the dose from ionizing radiation contributes to less than $50 \%$ of risk of cancer induction. The reason behind this is that there are other factors that contribute to the risk of cancer induction such as smoking which was excluded in the study. BEIR V report states that other major risk factors besides radiation (smoking, exposures to solvents, medical radiation exposures, chemotherapy, etc.) should be known for more accuracy in calculation. A case controlled study from cancer the Connecticut Tumor Registry showed an increased risk of $32.7 \%$ for second Ipsilateral lung cancer for women who smoked and were treated with breast radiotherapy [6].
The results from RHUH showed that for the majority of women treated for breast cancer with tangential wedged radiotherapy, RT did not play a significant role in the development of second primary breast cancer (REaverage $=1.00162$ ); however, young women with breast cancer had an elevated long-term risk of developing a Contralateral Breast cancer (REmax $=1.005$ for 30 years old woman). In addition this radiation risk was inversely related to age at exposure and was dose dependent. While The results from NBGUH showed that for the majority of women treated for breast cancer with radiotherapy, RT did not play a significant role in the development of second primary lung cancer (REaverage $=1.49$ ) or in spinal cord (REaverage $=1.362$ ); however, young women with breast cancer had an elevated longterm risk of developing a lung cancer ( $\mathrm{REmax}=1.58$ for 30 years old woman) and for spinal cord (REmax $=1.76$ for 30 years old woman). In addition this radiation risk was inversely related to age at exposure and was dose dependent. The major factors that may affect the incidence of secondary primary cancer induced by Breast cancer radiotherapy are the Radiation therapy process (safe radiotherapy practice) and the Estimated Risk / PTV. The percentage of compliance was $100 \%$ in treatment information transfer and the lowest was $65 \%$ in the planning phase as shown in Figure 3.

The major factors that may affect the secondary primary cancer induced by Breast cancer radiotherapy and errors threating the patient safety during radiotherapy process are categorized into 5 main factors: Environment, methods, machine, patient factor, and healthcare personnel. Using the 5M (Fishbone), see Figure 4 where the potential causes in increasing the risk of second malignancy after breast radiotherapy was analyzed.

\section{Conclusion}

This study reveals that the $85 \%$ of compliance in radiotherapy was due to safe practices. The calculated PC after breast radiotherapy for contralateral breast was $0.17 \%$ and PC for lung was $32 \%$, and for spinal cord was $24 \%$. While the PC in phantom measurements for the 
Ipsilateral lung was found to be $43.22 \%$ and PC for contralateral lung $3.099 \%$.

As a conclusion, many methods can be performed for evaluation of organ at risk doses in and out of the radiation field, and many preventive interventions can be applied to ensure patient safety during practices in radiotherapy process, the recommended preventive interventions should be taken that Lebanese ministry of health should add the radiotherapy audit as a specific chapter in accreditation standards and process $[7,8]$. Many studies proved that installation of new techniques during breast radiotherapy have decreased the risk of secondary cancers. These techniques such as Deep inspiration Breath Hold (DIBH), Proton Therapy, and Prophylactic Mammary Irradiation (PMI) may lower the radiation risks associated with breast treatment. In the anticipation of the development of improved algorithms of out-of-field dose calculation together with improved modeling of radio carcinogenesis throughout the range of delivered doses, the establishment of a rigorous Dosimetric framework is essential if future models are to be tested and retrospective dose estimates are to contribute usefully to epidemiological studies. The estimation of a second cancer risk is a difficult and extensive task and it is impracticable to simulate all possible treatment types and techniques for all patient geometries. In the meantime, every effort should be made to minimize the influence of factors that could potentially increase the risk of secondary cancers after radiotherapy. A lower total dose of radiation or non-radiation approach could be chosen for treatment whenever evidence supports the benefit without compromising tumor cure.

\section{Acknowledgements}

We would like to thank the health care professionals who were involved in the validation survey and clinical audits for this research project: NBGUH and $\mathrm{RHUH}$. Without their passionate participation and input, the clinical audit, and data collection could not have been successfully conducted.

\section{References}

1. Stovall M, Smith SA, Langholz BM, Boice JD, Shore RE, et al. (2008) Dose to the contralateral breast from radiotherapy and risk of second primary breast cancer in the wecare study. Int J Radiat Oncol Biol Phys 72: 1021-1030.

2. Mazonakisa M, Zacharopoulou F, Varveris H, Damilakis J (2008) Peripheral dose measurements for 6 and $18 \mathrm{MV}$ photon beams on a linear accelerator with multileaf collimator. Med Phys 35: 4396-4403.

3. Menzel H, Hrrison J (2011) Effective dose: A radiation protection quantity. Ann ICRP 41: 117-123.

4. Mazonakis $\mathrm{M}$, Varveris $\mathrm{H}$, Damilakis $\mathrm{J}$, Gourtsoyiannis N, Theoharopoulos N (2003) Radiation dose to conceptus resulting from tangential breast irradiation. Int J Radiat Oncol Biol Phys 55: 386-391.

5. Ng J, Shuryak I (2015) Minimizing second cancer risk following radiotherapy: Current perspectives. Cancer Manag Res 7: 1-11.

6. Martina OA, Yin X, Forrester HB, Sprung CN, Martin RF (2015) Potentia strategies to ameliorate risk of radiotherapy-induced second malignant neoplasms. Semin Cancer Biol 38: 65-76.

7. WHO (2008) Radiotherapy risk profile Technical Manual. Geneva, Switzerland

8. Oshaghi M, Sadeghi M, Mahdavi SR, Shirazi A(2013) Dosimetry of MammoSite applicator: Comparison between Monte Carlo simulation, measurements, and treatment planning calculation. J Cancer Res Ther 9: 224-229. 